Louisiana State University

LSU Digital Commons

Faculty Publications

Department of Physics \& Astronomy

10-15-2005

\title{
Semiclassical states, effective dynamics, and classical emergence in loop quantum cosmology
}

Parampreet Singh

Pennsylvania State University

Kevin Vandersloot

Pennsylvania State University

Follow this and additional works at: https://digitalcommons.Isu.edu/physics_astronomy_pubs

\section{Recommended Citation}

Singh, P., \& Vandersloot, K. (2005). Semiclassical states, effective dynamics, and classical emergence in loop quantum cosmology. Physical Review D - Particles, Fields, Gravitation and Cosmology, 72 (8)

https://doi.org/10.1103/PhysRevD.72.084004

This Article is brought to you for free and open access by the Department of Physics \& Astronomy at LSU Digital Commons. It has been accepted for inclusion in Faculty Publications by an authorized administrator of LSU Digital Commons. For more information, please contact ir@lsu.edu. 


\title{
Semiclassical states, effective dynamics, and classical emergence in loop quantum cosmology
}

\author{
Parampreet Singh ${ }^{1, *}$ and Kevin Vandersloot ${ }^{1,2, \dagger}$ \\ ${ }^{1}$ Institute for Gravitational Physics and Geometry, The Pennsylvania State University, 104 Davey Lab, University Park, \\ Pennsylvania 16802, USA \\ ${ }^{2}$ Max-Planck-Institut für Gravitationsphysik, Albert-Einstein-Institut, Am Mühlenberg 1, D-14476 Golm, Germany
}

(Received 15 July 2005; published 6 October 2005)

\begin{abstract}
We construct physical semiclassical states annihilated by the Hamiltonian constraint operator in the framework of loop quantum cosmology as a method of systematically determining the regime and validity of the semiclassical limit of the quantum theory. Our results indicate that the evolution can be effectively described using continuous classical equations of motion with nonperturbative corrections down to near the Planck scale below which the Universe can only be described by the discrete quantum constraint. These results, for the first time, provide concrete evidence of the emergence of classicality in loop quantum cosmology and also clearly demarcate the domain of validity of different effective theories. We prove the validity of modified Friedmann dynamics incorporating discrete quantum geometry effects which can lead to various new phenomenological applications. Furthermore the understanding of semiclassical states allows for a framework for interpreting the quantum wave functions and understanding questions of a semiclassical nature within the quantum theory of loop quantum cosmology.
\end{abstract}

DOI: 10.1103/PhysRevD.72.084004

PACS numbers: 04.60.Pp, 04.60.Kz, 98.80.Qc

\section{INTRODUCTION}

A fundamental input of loop quantum gravity (LQG) [1] to our understanding of quantum spacetime is that it is inherently discrete and spatial geometry is quantized. Quantum features of spacetime become evident in the regime of very high curvature whereas continuous spacetime emerges as a large eigenvalue limit of quantum geometry. Perhaps the most interesting avenue to explore this idea is in cosmological Friedmann-Robertson-Walker (FRW) spacetimes in the regime of high curvature and small volume such that quantum gravitational effects are expected to be dominant with the possibility of potentially observable signatures. It is a fundamental question in any viable model of LQG as to the scale at which the classical picture can be recovered and where precisely we expect to see modifications to the classical Friedmann dynamics. Since tools to address these issues are still under development [2], it is an open question whether the picture suggested by LQG holds for our Universe.

To construct a FRW model within the field theoretic framework of LQG would be quite difficult. Thus much progress has been made by restricting the model to a minisuperspace quantization known as loop quantum cosmology (LQC). In this simplified setting fundamental questions can be answered directly with explicit calculations and the physical consequences can be explored. As in LQG, the underlying geometry in LQC is discrete and the scale factor operator has discrete eigenvalues. Quantum dynamics is governed by a discrete difference equation which leads to a nonsingular evolution through the classical big bang singularity $[3,4]$. This important result can be

\footnotetext{
*Electronic address: singh@gravity.psu.edu

†Electronic address: kfvander@gravity.psu.edu
}

traced directly to the discrete nature of quantum geometry. A related and important feature of LQC is the modification to the behavior of the eigenvalues of the inverse scale factor below a critical scale factor $a_{*}$. Unlike in the classical regime, eigenvalues of the inverse scale factor operator become proportional to positive powers of the scale factor for $a<a_{*}$. These considerations have led to an effective description of the evolution of the Universe, where the standard Friedmann dynamics receives modifications from the inverse scale factor eigenvalues below $a_{*}$. Hence it has been assumed that classical emergence occurs above $a_{*}$ and that the Universe can still be described in terms of continuum dynamics below this scale.

The effective modified Friedmann dynamics leads to various interesting phenomenological effects (see Ref. [5] for a recent review). For example, it has been demonstrated that effective dynamics leads to a phase of kinetic dominated superinflation [6] which can provide correct initial conditions for conventional chaotic inflation [7-10], thus making inflation more natural. The loop quantum phase has been shown to alleviate the problem of inflation in closed models [11] and inflation without scalar field [12]. It is interesting to note that effects of a superinflationary phase prior to the conventional potential driven inflation may leave observable signatures at large scales in the cosmic microwave background [8]. Apart from effects in the early Universe, the effective dynamics promises to resolve various cosmological singularities, for example, the big crunch [13-16] and brane collision singularity [17]. Application of LQC techniques has also shown to yield nonsingular gravitational collapse scenarios with associated observable signatures $[18,19]$.

Although LQC phenomenology leads to various potentially observable effects, it should be noted that strictly speaking these investigations are not based directly on the 
quantum theory of LQC, but rather on heuristically motivated effective continuous equations of motion. Beyond heuristic ideas it is not clear as to the domain of validity of the effective theory and where the continuous effective description breaks down. Thus it merits a more careful derivation of the effective picture directly from the quantum theory and difference equation. In the absence of any such quantitative proof, the above assumptions on the existence of the modified dynamics below $a_{*}$ appear $a d$ hoc. These issues have been noted before and in fact phenomenological constraints on the domain of validity have also been discussed [9,20], but in the absence of any comparison with the underlying quantum evolution, such investigations are far from being complete.

A further issue less emphasized previously pertains to the fact that LQC predicts a discrete difference equation as opposed to the continuous Wheeler-DeWitt equation of standard quantum cosmology. It is thus important to determine under what conditions the discreteness can modify the dynamics and play an important role phenomenologically. An important question is whether the continuum picture breaks down in the regime where discreteness effects are important and if not how can the dynamics be described in terms of an effective continuous picture.

The aim of this paper is to answer these questions systematically through the application of the full quantum features of LQC. To this end we will study the model of a homogeneous and isotropic universe with matter coupled in the form of a massless scalar field. The main difficulty in describing dynamics within LQC pertains to the oft mentioned problem of time in quantum cosmology [21]. The problem can however be overcome by treating the scale factor as a clock variable and considering the evolution of the scalar field. Thus the semiclassical states constructed will consist of sharply peaked wave packets centered around a value of the scalar field at a particular scale factor. The states can be evolved forward (or backward) using the difference equation and the trajectory can be compared with that from classical Friedmann or effective dynamics. All of the physics is captured in this picture without resorting to an external time. This allows us to directly verify the semiclassical limit of LQC and determine where the continuum picture breaks down. The results will validate the effective continuous picture incorporating the inverse volume modifications. Furthermore, we will also test and verify previously proposed effective continuous equations that include modifications associated with the discreteness effects. We will indicate with the quantum evolution the conditions under which the discreteness effects play an important role.

\section{CLASSICAL THEORY AND THE QUANTUM CONSTRAINT}

The model we will investigate is composed of a homogeneous and isotropic universe with zero spatial curvature and matter in the form of a massless scalar field. The classical phase space is parametrized by four quantities: the connection $c$, the triad $p$, the scalar field $\phi$, and the scalar field momentum $P_{\phi}$ which satisfy the following Poisson bracket relations:

$$
\{c, p\}=\frac{1}{3} \kappa \gamma, \quad\left\{\phi, P_{\phi}\right\}=1 .
$$

Here $\gamma$ is the Barbero-Immirzi parameter whose value can be fixed to 0.2375 by black hole thermodynamics [22] and $\kappa=8 \pi G$. We will work with $c=\hbar=1$ and Planck length $l_{\mathrm{P}}=\sqrt{G}$. Note that formulas in LQC typically are written in terms of the modified Planck length $l_{\mathrm{P}}=\sqrt{8 \pi} \bar{G}$, hence our formulas will appear slightly modified from previous works.

The gravitational variables $c$ and $p$ encode the curvature and geometry, respectively, of the gravitational field which can be seen in their relation to the standard metric variables

$$
|p|=a^{2}, \quad c=\gamma \dot{a} .
$$

Governing the dynamics is the Hamiltonian constraint which, in terms of $c$ and $p$ variables, is given by

$$
H=-\frac{3}{\kappa \gamma^{2}} \operatorname{sgn}(p) \sqrt{|p|} c^{2}+\frac{1}{2} a^{-3} P_{\phi}^{2} .
$$

The equations of motion are derived through the vanishing of the Hamiltonian constraint $H=0$, and the Hamiltonian equations $(\dot{x}=\{x, H\}$ for any phase space variable $x)$. For the scalar field momentum, the Hamiltonian equations immediately imply that $P_{\phi}$ is constant in time. The Hamiltonian equations for $p$ and $\phi$ along with the vanishing of the Hamiltonian constraint give their time dependence

$$
\dot{p}= \pm \sqrt{\frac{2 \kappa}{3}} \frac{P_{\phi}}{a}, \quad \dot{\phi}=a^{-3} P_{\phi}
$$

which can be integrated to give the time evolution of both $p$ and $\phi$. The standard Friedmann equation can be derived by combining these equations, eliminating $P_{\phi}$, and using the relation between $p$ and $a$ to get

$$
\left(\frac{\dot{a}}{a}\right)^{2}=\frac{\kappa}{6} \dot{\phi}^{2}
$$

Our goal is to compare the trajectories of quantum wave packets in LQC with the classical equations of motion (4). The difficulty lies in the lack of any $\partial / \partial t$ term in the difference equation that governs the behavior of the wave packets. Thus it is impossible within LQC to consider a wave packet peaked around some value of $p$ and $\phi$ and evolve it forward in time to compare with the classical trajectory. This is the "problem of time" in quantum cosmology [21]. The origin of this difficulty can be understood even at the classical level; namely, that the classical equations of motion are not unique since the lapse is a freely specifiable function which we have implicitly fixed 
to one to arrive at (4). The trajectory $p(t)$ itself has no physical meaning since we can reparametrize $t$ to get a different trajectory. Physically, an observer could never measure the value of $t$ by measuring $p, c, \phi$, and $P_{\phi}$.

A solution to the problem, as noted by various authors [23], is to notice that while, for instance, $p(t)$ and $\phi(t)$ by themselves have no physical meaning, the correlation between $p$ and $\phi$ for a given value of $t$ is a physically meaningful statement. The correlations are invariant under reparametrizations of time. These correlations can be determined by deparametrizing the classical equations to remove the reference to $t$. For the model under consideration, we arrive at a differential equation governing the evolution of the scalar field as a function of the scale factor by noting $d \phi / d a=\dot{\phi} / \dot{a}$ which from the Friedmann equation gives

$$
\frac{d \phi}{d a}= \pm \sqrt{\frac{6}{\kappa a^{2}}} .
$$

Integrating this we find that the scalar field evolves as

$$
\phi_{\mathrm{cl}}(a)= \pm \sqrt{\frac{6}{\kappa}} \log (a)+C
$$

where $C$ is a constant. The time parameter $t$ showing up in the classical equations of motion can now be seen as an arbitrary parametrization of the correlations between the physical quantities given in (7).

To recover a notion of dynamics without any explicit reference to time in our model we can choose the scale factor to play the role of a physical clock since it is a monotonically increasing function as indicated in Eq. (4). While on general grounds it is not necessary to interpret the quantum theory by singling out a clock variable, in our model it proves useful for interpreting our results in terms of a "time evolution" of the scalar field given in Eq. (7). Thus we can consider wave packets peaked around a value of the scalar field at a given scale factor and then the difference equation of LQC will determine the trajectory of the wave packet for different values of the scale factor from which we can determine how the scalar field evolves with respect to the scale factor.

With an understanding of the classical framework we can now turn to the loop quantization of the model. In the framework of Dirac quantization used in LQG and LQC the physical quantum states are annihilated by the Hamiltonian constraint (3) represented as an operator. The gravitational side of the constraint when quantized using loop techniques leads to a partial difference equation [4]. The matter term is quantized as

$$
\hat{H}_{\phi}=\frac{1}{2} \hat{a}^{-3}{\hat{P_{\phi}}}^{2}=-\frac{1}{2} d_{J} \frac{\partial^{2}}{\partial \phi^{2}}
$$

with $d_{J}$ being the LQC quantized eigenvalues of the inverse volume operator. The key feature of the $d_{J}$ is that it is a bounded function with maxima at a characteristic scale factor $a_{*}=\sqrt{\left(8 \pi \gamma J \mu_{0}\right) / 3} l_{\mathrm{P}}$. The inverse volume eigenvalues are labeled with the quantum ambiguity parameter $J$ which arises from the fact that the inverse volume operator is computed by tracing over SU(2) holonomies in an irreducible spin $J$ representation [24]. Physically, below the scale factor $a_{*}$ determined by $J$, the inverse volume eigenvalues are suppressed in contrast to the classical inverse volume which diverges for small $a . \mu_{0}$ is an additional quantum ambiguity parameter which is heuristically related to the smallest eigenvalue of area operator in LQG [4] (which fixes its value to $\sqrt{3} / 4$ ). The eigenvalues $d_{J}$ can be approximated as $d_{J}(a)=D(a) a^{-3}$ [24] with

$$
\begin{aligned}
D(a)= & (8 / 77)^{6} q^{3 / 2}\left\{7\left[(q+1)^{11 / 4}-|q-1|^{11 / 4}\right]\right. \\
& \left.-11 q\left[(q+1)^{7 / 4}-\operatorname{sgn}(q-1)|q-1|^{7 / 4}\right]\right\}^{6},
\end{aligned}
$$

where $q \equiv a^{2} / a_{*}^{2}$. For $a>a_{*}$ we have $D(a) \approx 1$ and we recover the classical expression for the inverse volume eigenvalues $d_{J}(a) \approx a^{-3}$.

The constraint equation satisfied by the physical states $\psi_{\mu}(\phi)$ becomes

$$
\begin{aligned}
& \frac{3}{4 \kappa \gamma^{2} \mu_{0}^{2}}\left[s\left(\mu+4 \mu_{0}\right) \psi_{\mu+4 \mu_{0}}-2 s(\mu) \psi_{\mu}\right. \\
& \left.\quad+s\left(\mu-4 \mu_{0}\right) \psi_{\mu-4 \mu_{0}}\right]-\frac{1}{2} d_{J}(\mu) \frac{\partial^{2} \psi_{\mu}}{\partial \phi^{2}}=0
\end{aligned}
$$

where the parameter $\mu$ is an eigenvalue of the scale factor operator and volume operator given by

$$
a=\sqrt{\frac{8 \pi \gamma|\mu|}{6}} l_{\mathrm{P}}, \quad V_{\mu}=\left(\frac{8 \pi \gamma|\mu|}{6}\right)^{3 / 2} l_{\mathrm{P}}^{3}
$$

and the function $s(\mu)=\left[2 /\left(8 \pi \gamma \mu_{0} l_{\mathrm{P}}^{2}\right)\right]\left(V_{\mu+\mu_{0}}-V_{\mu-\mu_{0}}\right)$ can be shown to be equal to $\sqrt{p}=a$ for large volumes [20]. We note that this difference equation is derived from a non-self-adjoint constraint operator. One can construct a self-adjoint constraint $[25,26]$ and derive the corresponding difference equation, however we do not expect our results to be modified significantly. The difference equation can also be seen as a discrete approximation of the second order hyperbolic Wheeler-DeWitt equation

$$
\frac{\kappa}{3} \frac{\partial^{2}}{\partial p^{2}}(\sqrt{p} \psi(p, \phi))-\frac{1}{2} d_{J} \frac{\partial^{2} \psi(p, \phi)}{\partial \phi^{2}}
$$

obtained by quantizing $\hat{c}=i \frac{1}{3} \gamma \kappa \partial / \partial p$. It has been shown in Ref. [27] that wave packets which satisfy the WheelerDeWitt equation (though for a different factor ordering than that given here and without $d_{J}$ corrections) follow the classical trajectory given in Eq. (7). The constraint equation (10) differs radically from the Wheeler-DeWitt equation by the fact that it is a discrete difference equation and by the presence of the inverse volume eigenvalues $d_{J}$. 
It is our goal now to determine the precise effects of these differences in the trajectory of the wave packets.

Attempts have been made to describe the new features of the quantum theory of LQC within an effective continuous theory. The very first attempts simply replaced the inverse volume in the classical equations of motion with the eigenvalues $d_{J}$ [6]. Many of the phenomenological investigations are based on this effective framework. The effective equations have been generalized both through a path integral framework [20] and a WKB analysis [28,29] to include effects arising from the fundamental discreteness of the theory. The effective framework can be described in terms of an effective Hamiltonian constraint given by (additional terms arise in WKB analysis, which are not considered here)

$$
H_{\text {eff }}=-\frac{3}{\kappa \gamma^{2} \mu_{0}^{2}} \sqrt{p} \sin ^{2}\left(\mu_{0} c\right)+\frac{1}{2} d_{J}(a) P_{\phi}^{2}
$$

where the $\sin ^{2}\left(\mu_{0} c\right)$ modifications can be understood as discreteness corrections from the difference equation. Most phenomenological investigations so far have ignored the discreteness corrections by assuming $\mu_{0} c \ll 1$ and $\sin \left(\mu_{0} c\right) \approx \mu_{0} c$ whence the modified Friedmann equation becomes

$$
\left(\frac{\dot{a}}{a}\right)^{2}=\frac{\kappa}{3} \rho_{m}=\frac{\kappa}{3} \frac{\dot{\phi}_{\mathrm{eff}}^{2}}{2 D(a)}
$$

where $\rho_{m}=\frac{1}{2} d_{J} P_{\phi}^{2} / a^{3}=\frac{1}{2} D(a) P_{\phi}^{2}$ and we have used $\dot{\phi}_{\text {eff }}=d_{J} P_{\phi}$ acquired from the Hamiltonian equations. It is then straightforward to deparametrize the equations of motion to obtain

$$
\frac{d \phi_{\mathrm{eff}}}{d a}= \pm \sqrt{\frac{6 a d_{J}(a)}{\kappa}} .
$$

For $a<a_{*}, d_{J}(a)$ is proportional to positive powers of the scale factor which leads to radical modifications of the $\phi_{\text {eff }}(a)$ trajectory compared to the one obtained classically. For $a$ larger than $a_{*}$, we have $d_{J}(a) \approx a^{-3}$ and we recover the classical behavior of the scalar field given in Eq. (7).

In our analysis we would also like to consider the discreteness corrections from the gravitational side of the constraint. From the vanishing of the effective constraint (13) it is clear that the discreteness corrections become relevant when the matter term becomes large. More precisely when the matter density $\rho_{m}$ is on the order of a critical density $\rho_{\text {crit }} \equiv 3 / \kappa \mu_{0}^{2} \gamma^{2} a^{2}$, the corrections are appreciable [20]. The equations of motion can be calculated from the effective constraint (13) and deparametrized to give

$$
\begin{aligned}
\frac{d \phi_{\mathrm{eff}}}{d a} & = \pm \sqrt{\frac{6 a d_{J}}{\kappa} \frac{1}{\left[1-\left(\kappa \gamma^{2} \mu_{0}^{2} d_{J} P_{\phi}^{2} / 6 a\right)\right]}} \\
& = \pm \sqrt{\frac{6 a d_{J}}{\kappa} \frac{1}{\left(1-\rho_{m} / \rho_{\text {crit }}\right)}}
\end{aligned}
$$

whence it is clear that the modified equations of motion in Eq. (15) are recovered when $\rho_{m} \ll \rho_{\text {crit }}$. The Friedmann equation with discrete quantum corrections can be obtained from the above equation and is given by [20]

$$
\left(\frac{\dot{a}}{a}\right)^{2}=\frac{\kappa}{3} \rho_{m}-\frac{1}{9} \kappa^{2} \gamma^{2} \mu_{0}^{2} a^{2} \rho_{m}^{2} .
$$

For simplicity we will refer to effective theories described by Eqs. (15) and (16) as ET-I and ET-II, respectively.

As emphasized earlier, the use of these effective continuous equations requires a more careful consideration. Most phenomenological investigations have so far assumed that the effective continuous equations remain valid even near the Planck regime, in particular, until the fundamental step size of the difference equation $4 \mu_{0}$ which corresponds to a scale factor of $a_{0} \approx \sqrt{16 \pi \gamma \mu_{0} / 3} l_{\mathrm{P}}$. There-

fore it is crucial to determine under what conditions the effective equations hold and the phenomenological predictions can be trusted. It is important to determine the scale at which discrete quantum geometric effects play a prominent role and influence dynamics. We can then answer the question as to what scale the continuum spacetime arises and classicality emerges. We can also determine if further corrections arise from pure quantum effects.

\section{COHERENT STATE EVOLUTION AND QUANTUM DYNAMICS}

Our aim is to compare the classical and effective theories with the evolution from the difference equation, and thus for simplicity we do not consider the evolution beyond the classical singularity. The difference equation (10) is sufficiently complicated such that an analytic solution is not available. We will thus compute the solutions numerically. In our method we consider a semiclassical state at a large initial scale factor and evolve it backward toward the singularity using the difference equation. As a semiclassical state we consider a Gaussian wave packet sharply peaked around $\phi=0$ and some classical $P_{\phi}$ at the scale factor $a_{\text {init }} \gg a_{*}$,

$$
\psi_{a_{i}}(\phi)=\exp \left(i P_{\phi} \phi\right) \exp \left(-\phi^{2} / 2 \sigma^{2}\right)
$$

with a spread $\Delta \phi=\sigma$. Since the difference equation is second order in $\mu$, to find a physical solution we must specify initial conditions at $\mu_{\text {init }}$ [determined from $a_{\text {init }}$ using Eq. (11)] and $\mu_{\text {init }}-4 \mu_{0}$. The difference equation then gives us the wave function at $\mu_{\text {init }}-8 \mu_{0}$ which serves to determine the wave function at the next step 
and so on, thus yielding us evolution of the initial Gaussian. The scalar field trajectory $\phi(a)$ will then be obtained from the peak of the semiclassical state (we will comment on the validity of this in the discussion).

Given the Gaussian initial condition, exact solutions can be computed numerically by assuming the form

$$
\psi_{\mu}(\phi)=\exp \left(i P_{\phi} \phi\right) \exp \left(-\phi^{2} / 2 \sigma^{2}\right) \sum_{n=0}^{\infty} C_{n}(\mu) \phi^{n} .
$$

With the above ansatz, the partial difference equation is reduced to a difference equation for the coefficients $C_{n}(\mu)$ which can be solved numerically (the ansatz avoids computation of finite differences of $\left.\partial^{2} \psi_{\mu} / \partial \phi^{2}\right)$. The initial condition is then simply $C_{n}\left(\mu_{\text {init }}\right)=\delta_{n 0}$ for some large $\mu_{\text {init }}$. We are left to specify the wave function at $\mu_{\text {init }}-$ $4 \mu_{0}$. This choice does not affect our results appreciably. We specify the initial condition by analogy with the KleinGordon equation where an arbitrary solution is the sum of positive and negative frequency solutions [which correspond to the \pm solutions of Eqs. (7), (15), and (16)]. We can then tune the initial conditions to pick out one of the two solutions.

For the given initial coherent state peaked at $\phi=0$ and $P_{\phi}$ we can also evaluate the classical and effective trajectories from ET-I and ET-II and compare them with the trajectory of the peak of the coherent state. For larger values of $J, a_{*}$ increases and we thus expect to see deviations from the classical dynamics at larger scales (yet still below $a_{*}$ ). The deviations from ET-II are expected when the matter density approaches the critical value. We can test these corrections by choosing a large initial value of $P_{\phi}$. Furthermore, since the fundamental discrete step is of size $4 \mu_{0}$ we do not expect classical or effective theories to be valid for $\mu \lesssim 4 \mu_{0}$. We now discuss some representative cases from our numerics:

(i) $J=1 / 2, P_{\phi}=10 l_{p}$ : This case corresponds to the smallest value of $J$ and a small value of $P_{\phi}$ with $\mu_{\text {init }}=$ 200. The matter density remains small compared to the critical value which ensures that differences between ET-I and ET-II are negligible throughout the evolution. Since $J=1 / 2$, we have $a_{*}<a_{0}$ which implies that the effective dynamics agrees with the classical dynamics for all scale factors. The evolution of the coherent state via the quantum difference equation is shown in Fig. 1. The coherent state is sharply peaked at $\mu_{\text {init }}$ and evolves toward $\mu=0$ without losing its semiclassical character and retaining its sharp peak. The trajectory of the peak is compared to the classical and effective theories in Fig. 2. The classical and effective theories are in very good agreement with quantum theory until the smallest nonzero value of $\mu$. It is clear that for this choice of parameters, the classical evolution can be trusted until the first step in the quantum evolution before the classical singularity, i.e. until $a_{0}$. Below $a_{0}$ the classical evolution would lead to a blow up of $\phi$ resulting in a singularity whereas the evolution is nonsingular with the

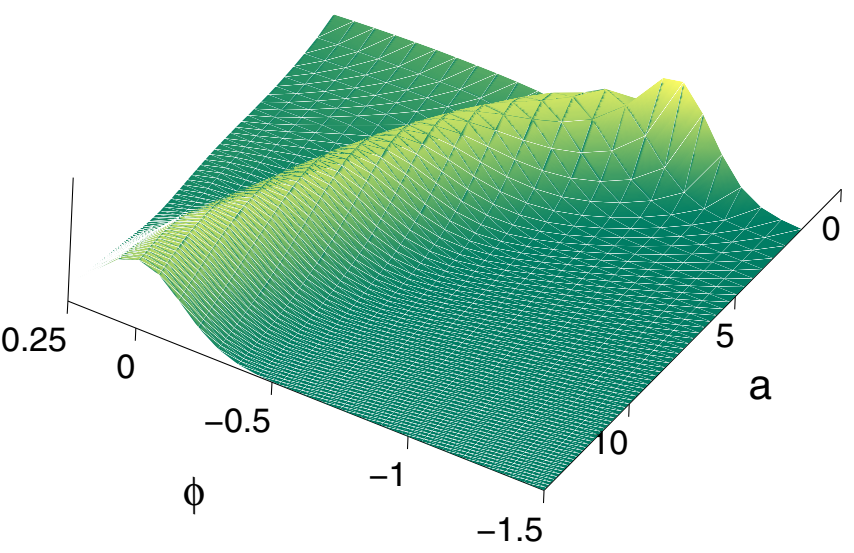

FIG. 1 (color online). Evolution of the coherent state for $J=$ $1 / 2$ and $P_{\phi}=10 l_{p}$. The coherent state remains sharply peaked and follows the classical trajectory given by Eq. (7). Amplification at scales close to $a=0$ are due to discrete quantum effects.

quantum difference equation. For the chosen value of parameters, classicality and continuum thus emerge as soon as we consider a scale factor greater than $a_{0}$.

(ii) $J=500, P_{\phi}=100 l_{p}$ : Here we start the evolution at $\mu_{\text {init }}=350$ which corresponds to an initial scale factor twice $a_{*}$. In this case $a_{*}>a_{0}$ and we expect a region where the classical theory breaks down and dynamics can be approximated by the effective theory. We have chosen $P_{\phi}$ and $\mu_{i}$ in such a way that differences between ET-I and ET-II are negligible even for very small $\mu$. The results are plotted in Fig. 3. It is evident that the classical theory departs from the quantum evolution at a larger scale factor as compared to the case of $J=1 / 2$. The scale at which

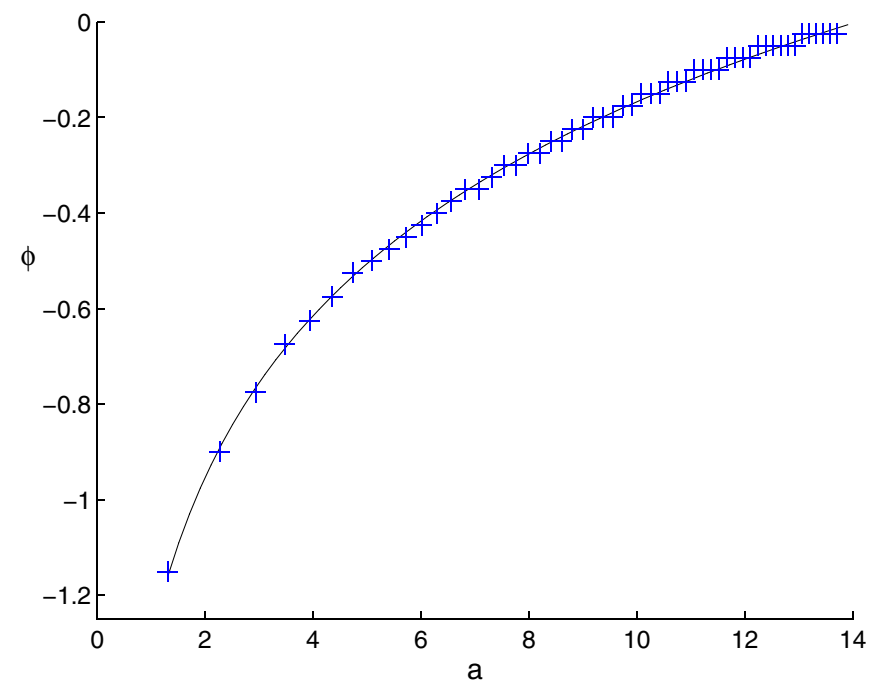

FIG. 2 (color online). The trajectory of the peak of the coherent state $(+$ line) is compared with classical theory (solid line) for $J=1 / 2$ and $P_{\phi}=10 l_{p}$. The classical trajectory agrees extremely well with the quantum curve until the smallest discrete step in the scale factor. 
PARAMPREET SINGH AND KEVIN VANDERSLOOT

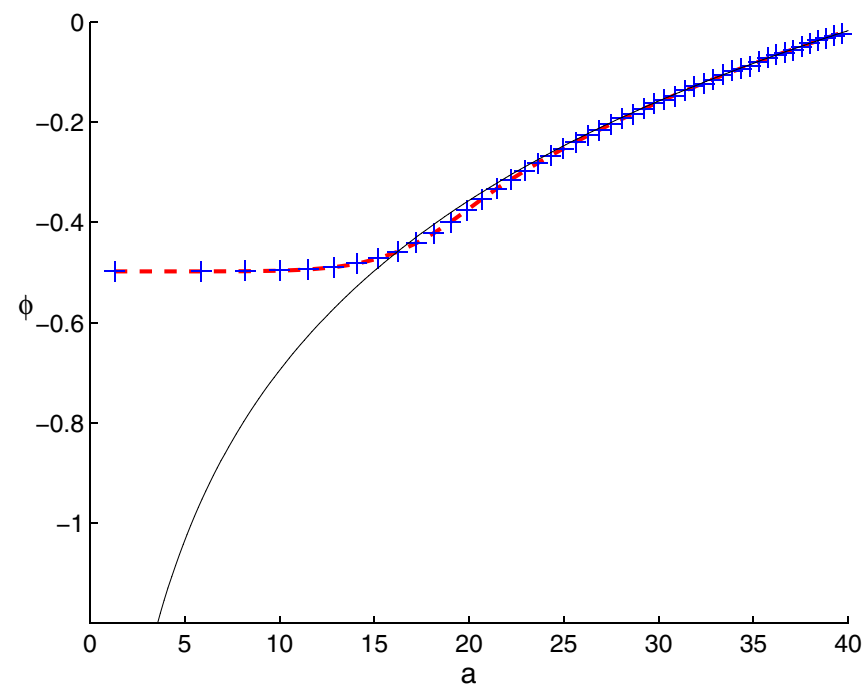

FIG. 3 (color online). Comparison of evolution via the quantum constraint (+ line), ET-I and ET-II (dashed line), and classical theory (solid line) for $J=500, P_{\phi}=100 l_{p}$. Classical theory breaks down for $a<a_{*}$, whereas ET-I and ET-II are valid until $a_{0}$.

departure becomes significant is $a_{*}$. It should be noted that ET-I and ET-II agree with the quantum difference evolution until the smallest step size in the scale factor. Thus, for large $J$ and reasonably chosen $P_{\phi}$ such that $\rho_{m} \ll \rho_{\text {crit }}$ during evolution, ET-I and ET-II can be trusted to the smallest allowed scale factor $a_{0}$. Unlike case (i), classicality emerges for scale factors $a>a_{*}$, however continuum spacetime is a good approximation to discrete quantum geometry for $a>a_{0}$.

(iii) $J=500, P_{\phi}=1600 l_{p}$ : As in case (ii) we start the evolution from $\mu_{\text {init }}=350$. However, we now choose $P_{\phi}$ such that the matter density becomes on the order of the critical value for scale factors greater than $a_{*}$. We thus expect significant departure between the quantum evolution from classical theory and ET-I for larger scale factors compared to those in case (ii). The results are shown in Fig. 4. The ET-II corrections to the Friedmann dynamics become significant at $a \sim 20 l_{p}$ where both the classical and ET-I dynamics start to disagree with the quantum evolution. ET-II which incorporates these corrections matches very well the coherent state evolution until $a_{0}$. The inset of the figure shows the evolution of the matter density with the scale factor. The dashed line represents the critical density $\rho_{\text {crit }}$ and it is clear that near $a \sim 20 l_{p}$, the matter density becomes on the order of the critical value and precisely there the deviations due to ET-II arise.

The picture which emerges from these cases is confirmed in various other numerical studies we performed. All of the numerical results can be broadly classified in the above three cases. In summary our results show:

(i) The classical evolution matches very well with the quantum evolution until very small scale factors.
PHYSICAL REVIEW D 72, 084004 (2005)

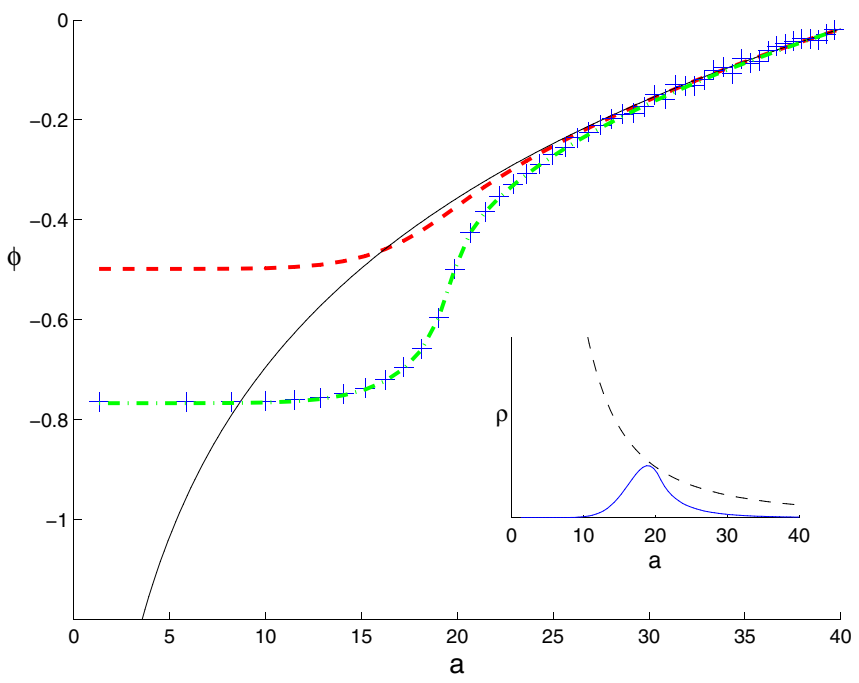

FIG. 4 (color online). Evolution for $J=500, P_{\phi}=1600 l_{p}$. The classical (solid line) and ET-I trajectory (dashed line) deviate from quantum evolution (+ line) for scale factors at which perturbative corrections become significant. ET-II agrees with evolution from the quantum constraint until $a_{0}$. Inset: ET-II corrections become significant when the matter density (solid line) approaches the critical density (dashed line).

For small $J$ and small $P_{\phi}$, the classical theory can even be trusted until $a_{0}$. For large $J$ and/or large $P_{\phi}$, departures occur at larger scale factors.

(ii) The effective theories with appropriate corrections are very good approximations to difference equations until scale $a_{0}$. With suitable choice of initial conditions such that the gravitational corrections of ET-II are negligible, various phenomenological applications based on ET-I are trustworthy.

(iii) Since effective theories are good approximations for $a>a_{0}$, the assumption that continuum spacetime emerges above $a_{0}$ proves reasonable. Emergence of classicality, however, depends on various factors. For small $J$ it may occur for $a \sim$ $a_{0}$ (if $P_{\phi}$ is not large). For large $J$ it emerges at larger values of the scale factor.

\section{DISCUSSION}

Let us summarize the main results presented in this work. We were able successfully to construct semiclassical states within the discrete framework of LQC which were represented as localized wave packets that for large volumes followed the classical trajectory. This can be viewed as a systematic demonstration that LQC has the correct semiclassical limit while leading to modifications in the high curvature regime of the Universe. Our results indicate that the discrete nature of the Universe predicted by LQC can be adequately described in terms of an effective continuous picture down to the Plank scale. Since the quantum theory of LQC predicts discrete steps of the order of the 
Planck length, it can safely be said that the continuous picture breaks down below the Planck scale. The surprising conclusion that follows from our results is that the continuous emergence occurs very near the Planck scale. This does not imply, however, that above the Planck scale we recover the standard Friedmann dynamics. The emergence of the classical spacetime depends on choice of $P_{\phi}$ and $J$ in the initial configuration and following our procedure the scale of classical emergence can be found in a straightforward way.

We have also demarcated explicitly the domain of validity of effective descriptions ET-I and ET-II. The former was based on including modifications to the inverse scale factor in the standard Friedmann dynamics, whereas the latter also includes the modifications pertaining to large extrinsic curvature. For the choice of initial conditions such that modifications due to extrinsic curvature are negligible, ET-I serves as a good effective theory. Most of the phenomenological applications investigated so far in LQC fall into this category. However, for generic initial conditions ET-II is a more faithful description of the quantum theory of LQC. This is the new phenomenological input in LQC and opens a new avenue for various applications.

Let us compare our results with earlier proposals for effective dynamics. In one approach kinematical coherent states are constructed and then an effective Hamiltonian constraint is calculated from the expectation value of the constraint operator acting on these states [25]. The relation between the kinematical coherent states in that work and the coherent states considered in this paper is not clear since the kinematical coherent states are not annihilated by the Hamiltonian constraint operator and are thus not physical states. There is some evidence that a technique known as group averaging to "project" kinematical states into physical ones (and in the process provide a physical inner product which we discuss later) will project the kinematical coherent states into physical ones with similar properties [30], thus providing a connection between their work and ours. Further, in the kinematical coherent state approach additional corrections are calculated which depend on the spread of the coherent state. Effects of these corrections have not been considered in this paper. Interestingly, the obtained modifications to the classical equations are very similar in both approaches and a more detailed analysis is needed to make a complete comparison.

A second approach has been to consider a WKB approximation for solutions to the difference equation and from that extract an effective Hamiltonian constraint $[28,29]$. Through this technique one calculates the same effective Hamiltonian constraint as that given in Eq. (13) plus an additional term denoted by the quantum geometry potential. Our results of testing ET-II are directly applicable to this approach and suggest that phenomenological applications based on WKB analysis occurring at scale factors greater than $a_{0}$ due to inclusion of higher order terms in $\sin \left(\mu_{0} c\right)$ expansion [as in Eq. (13)] seem trustworthy. Since the evolution occurs with discrete steps of the order of $a_{0}$, any effect based on continuum dynamics below this scale requires further justification. In this work we have not tested the effects of additional potential in the WKB Hamiltonian constraint. A careful and detailed analysis is required to understand the significance of the derived potential in this approach.

Finally another method has been to consider the evolution of kinematical coherent states by evolving them with the unitary operator acquired by exponentiating the Hamiltonian constraint operator [26]. The effect is to introduce a coordinate time parameter into the quantum theory. This is equivalent to gauge fixing the lapse to unity and then quantizing the remaining Hamiltonian as an unconstrained system which leads to a difference equation with a Schrodinger equation like the $\partial / \partial t$ term on the right-hand side. Performing this procedure, dynamics is easy to describe in terms of the coordinate time parameter now appearing in the difference equation. However, the framework presented in this paper has the advantage that the wave functions are physical states, i.e. those that satisfy the difference equation. This is a crucial feature which testifies to the validity of our results. Within the method considered here, various continuous effective equations of motion can be tested in a precise manner. Since explicit gauge fixing is involved in the analysis of Ref. [26], a comparison of those results with the ones obtained here is difficult.

Let us turn to an open issue in the program presented here. In extracting the trajectory of the scalar field from the wave functions we have used the peak of the wave packet. Properly done, the trajectory should be calculated as an expectation value of the operator $\hat{\phi}$ with a suitable probability measure provided by the physical inner product. A procedure for providing a physical inner product that is suitable for constrained systems like LQC is known as group averaging [31] and this technique has been explored in depth in a model of LQC with a cosmological constant in [32]. We can speculate as to the effect of using the correct probability measure by noting the similarity of the difference equation to the Klein-Gordon equation. The application of group averaging to the Klein-Gordon constraint yields a probability measure that is time independent and positive definite on the space of both positive and negative frequency solutions. It can be shown that when considering coherent states which are sharply peaked, the expectation value of the scalar field using the group averaging probability measure is approximately equal to the peak of the wave packet which is evidence that we do not expect the results of this paper to be significantly modified by using the correct probability measure. This does not rule out the possibility of corrections arising from the measure in the small volume regime where discreteness effects may play an important role in the physical inner product. 
This work opens the possibility for further quantum applications of semiclassical nature. Apart from previously considered phenomenological applications, it will be interesting to consider, for instance, anisotropic models where one of the geometrical degrees of freedom can serve as a clock and semiclassical wave functions can be constructed similarly. Furthermore the dynamics of ET-II suggests the existence of bouncing and recollapsing phases when the matter density becomes large. This also opens the possibility for the presence of tunneling regions, the description of which would require the understanding of the quantum evolution of semiclassical states. Additional applications include investigations of the evolution through the singularity and the relation to pre-big-bang scenarios.

\section{ACKNOWLEDGMENTS}

We would like to thank Abhay Ashtekar for useful discussions and helpful comments, and Martin Bojowald for a careful reading of the manuscript and helpful comments. This work was supported by the Eberly research funds of Penn State and NSF Grant No. PHY-0090091 and No. PHY-0354932.
[1] T. Thiemann, Lect. Notes Phys. 631, 41 (2003); A. Ashtekar and J. Lewandowski, Classical Quantum Gravity 21, R53 (2004); C. Rovelli, Quantum Gravity, Cambridge Monographs on Mathematical Physics (Cambridge University Press, Cambridge, England, 2004).

[2] J. Brunnemann and T. Thiemann, gr-qc/0505032.

[3] M. Bojowald, Phys. Rev. Lett. 86, 5227 (2001).

[4] A. Ashtekar, M. Bojowald, and J. Lewandowski, Adv. Theor. Math. Phys. 7, 233 (2003).

[5] M. Bojowald, gr-qc/0505057.

[6] M. Bojowald, Phys. Rev. Lett. 89, 261301 (2002).

[7] M. Bojowald and K. Vandersloot, Phys. Rev. D 67, 124023 (2003).

[8] S. Tsujikawa, P. Singh, and R. Maartens, Classical Quantum Gravity 21, 5767 (2004).

[9] M. Bojowald, J.E. Lidsey, D. J. Mulryne, P. Singh, and R. Tavakol, Phys. Rev. D 70, 043530 (2004).

[10] G. Date and G. M. Hossain, Phys. Rev. Lett. 94, 011301 (2005).

[11] J. E. Lidsey, D. J. Mulryne, N. J. Nunes, and R. Tavakol, Phys. Rev. D 70, 063521 (2004).

[12] P. Singh, Classical Quantum Gravity 22, 4203 (2005).

[13] P. Singh and A. Toporensky, Phys. Rev. D 69, 104008 (2004).

[14] G. V. Vereshchagin, J. Cosmol. Astropart. Phys. 07 (2004) 013.

[15] D. J. Mulryne, N. J. Nunes, R. Tavakol, and J. E. Lidsey, Int. J. Mod. Phys. A 20, 2347 (2005).

[16] G. Date and G. M. Hossain, Phys. Rev. Lett. 94, 011302 (2005).

[17] M. Bojowald, R. Maartens, and P. Singh, Phys. Rev. D 70, 083517 (2004).

[18] M. Bojowald, R. Goswami, R. Maartens, and P. Singh, Phys. Rev. Lett. 95, 091302 (2005).

[19] R. Goswami, P. S. Joshi, and P. Singh, gr-qc/0506129.

[20] K. Vandersloot, Phys. Rev. D 71, 103506 (2005).

[21] K. Kuchar, in Proceedings of the 4th Canadian
Conference on "General Relativity and Relativistic Astrophysics", edited by G. Kunstatter, D. Vincent, and J. Williams (World Scientific, Singapore, 1992); C. J. Isham, Canonical Quantum Gravity and the Problem of Time, Integrable Systems, Quantum Groups and Quantum Field Theories, edited by L. A. Ibort and M. A. Rodriguez (Kluwer, London, 1993).

[22] A. Ashtekar, J. Baez, A. Corichi, and K. Krasnov, Phys. Rev. Lett. 80, 904 (1998); A. Ashtekar, J. C. Baez, and K. Krasnov, Adv. Theor. Math. Phys. 4, 1 (2000); M. Domagala and J. Lewandowski, Classical Quantum Gravity 21, 5233 (2004); K. A. Meissner, Classical Quantum Gravity 21, 5245 (2004).

[23] J. Barbour, Classical Quantum Gravity 11, 2853 (1994); J. Barbour, Classical Quantum Gravity 11, 2875 (1994); C. Rovelli, Phys. Rev. D 65, 124013 (2002); M. Montesinos, C. Rovelli, and T. Thiemann, Phys. Rev. D 60, 044009 (1999); M. Montesinos, Gen. Relativ. Gravit. 33, 1 (2001).

[24] M. Bojowald, Classical Quantum Gravity 19, 5113 (2002).

[25] J. Willis, Ph.D. thesis, The Pennsylvania State University, 2004.

[26] M. Bojowald, P. Singh, and A. Skirzewski, Phys. Rev. D 70, 124022 (2004).

[27] C. Kiefer, Phys. Rev. D 38, 1761 (1988).

[28] G. Date and G. M. Hossain, Classical Quantum Gravity 21, 4941 (2004).

[29] K. Banerjee and G. Date, Classical Quantum Gravity 22, 2017 (2005).

[30] A. Ashtekar, L. Bombelli, and A. Corichi, Phys. Rev. D 72, 025008 (2005).

[31] A. Ashtekar, J. Lewandowski, D. Marolf, J. Mourao, and T. Thiemann, J. Math. Phys. (N.Y.) 36, 6456 (1995); D. Marolf, gr-qc/0011112.

[32] K. Noui, A. Perez, and K. Vandersloot, Phys. Rev. D 71, 044025 (2005). 